\title{
Combined Therapy of Pioglitazone and Atorvastatin Alleviate Diabetes in Rats More Effectively than That of Mono Therapy
}

\author{
Hazrat Ali, A. F. M. Towheedur Rahman, Saiful Islam, Al Mamun, Shaheda Zannah, \\ A. H. M. Khurshid Alam, Aziz Abdur Rahman, Mamunur Rashid*
}

Department of Pharmacy, University of Rajshahi, Rajshahi, Bangladesh

Email: * mamun69jp@yahoo.com

Received 5 March 2014; revised 16 April 2014; accepted 12 May 2014

Copyright (C) 2014 by authors and Scientific Research Publishing Inc.

This work is licensed under the Creative Commons Attribution International License (CC BY). http://creativecommons.org/licenses/by/4.0/

\begin{abstract}
Present research was designated to investigate the hypoglycemic, hypolipidemic and antioxidant activity of the combination of pioglitazone and atorvastatin on long-term alloxan-induced diabetes rats (AIDRs). The experiments were carried out to determine blood glucose level, lipid profile, free radial scavenging activities, superoxide dismutase (SOD) and catalase in liver tissue. In addition, left ventricular (LV) hypertrophy and cardiomyocyte size were also determined by histological analysis. It was found that in short-term induction, pioglitazone significantly reduced the blood glucose level without having any considerable effect on lipid profile and antioxidant enzymes (SOD and catalase) in rats. On the other hand, atorvastatin significantly reduced total cholesterol (TC), triglyceride (TG) and low density lipoprotein cholesterol (LDL-C) with marked increase in the level of high density lipoprotein cholesterol (HDL-C) and improved activity of SOD and catalase enzymes. However, pathological changes of heart and pancreas were not observed after shortterm exposure to alloxan in rats. Long-term diabetes induction resulted in LV hypertrophy and prominent shrinkage of islets of Langerhans cells. Treatment with atorvastatin in combination with pioglitazone significantly reduced the LV hypertrophy, TC, TG and LDL level whereas they noticeably increased HDL level, DPPH (1,1-Diphenyl-2-picryl-hydrazyl) free radical scavenging activity, SOD and catalase activity with satisfactory recovery of Langerhans cells. The result demonstrated that combination therapy was more effective than that of mono-therapy for preventing diabetes with cardiovascular diseases (CVD) in rats.
\end{abstract}

\section{Keywords}

Diabetes with CVD, Pioglitazone, Atorvastatin, Combination Drugs

\footnotetext{
${ }^{*}$ Corresponding author.
}

How to cite this paper: Ali, H., et al. (2014) Combined Therapy of Pioglitazone and Atorvastatin Alleviate Diabetes in Rats More Effectively than That of Mono Therapy. Pharmacology \& Pharmacy, 5, 504-513. 


\section{Introduction}

The prevalence of diabetes has become an issue of great concern worldwide. It is considered as a leading degenerative disease in today's world, affecting approximately 15 million people [1]. According to the forecast of World Health Organization (WHO), this disease will be the seventh leading cause of death by 2030 [2]. The term, diabetes mellitus, signifies a set of metabolic disorders, having multiple etiologies, characterized by hyperglycemia which is attributed by the defect in insulin secretion, insulin action or both [3]. It has a drastic consequence on carbohydrate, lipid and protein metabolism, and ultimately leads to chronic hyperglycemia and abnormal lipid profile. Eventually, these abnormalities potentiate a series of secondary complications including polyurea, polyphasia, ketosis, retinopathy as well as cardiovascular diseases [4].

In diabetic patients, hyperglycemia boosts oxidative stress as an outcome of excess fatty acid release and subsequent activation of lipoxygenase and xanthine oxidase pathways [5]. Activated lipoxygenases [6] and xanthine oxidase [7] further promotes nitric oxide synthase (NOS) that switches a coupled state to an uncoupled state and generates superoxide anion [8] with decreased 5,6,7,8-tetrahydrobiopterin (BH4) or L-arginine. Dysfunctional mitochondrial electron-transport chain is another source of reactive oxygen species (ROS). The ROS increase the oxidation of the lipid (i.e. LDL) which was then ingested by the macrophage, responsible for plaque formation on the heart vessels and ultimately develops common cardiovascular diseases (CVDs) like atherosclerosis, left ventricular hypertrophy etc. [9].

Another study showed that ROS reduced bioavailability of nitric oxide (NO), leading to endothelial dysfunction. ROS also influence the activity of a number of cell signaling pathways, and ultimately lead to the changes in the expression of redox-sensitive genes that regulate cellular process involved in cellular apoptosis. This factor is most probably involved in the pathogenesis of cardiovascular diseases [10].

Moreover, long-term induction of diabetes without any lipid lowering drug accelerates the risk of CVD. Hence, combination therapy has been suggested as a useful anti-diabetic and hypolipidemic remedy due to their ability to restore the function of pancreatic tissues by causing an increase insulin output, to inhibit the intestinal absorption of glucose, to facilitate the metabolites in insulin dependent processes [11] and/or by their ability to inhibit the cholesterol biosynthetic pathway by inhibiting the enzyme HMG-CoA reductase [12]. Hence, treatment with combination therapy has an effect on reviving $\beta$-cells and restoring the fluctuation in glucose level and cholesterol biosynthetic pathway.

The current study is aimed to provide strong evidence, based on experimental studies carried out on the most effective and commonly used hypoglycemic drug (Pioglitazone) and lipid lowering drug (Atorvastatin) alone and in combination so that we can treat our diabetic patients along with their cardiac diseases. Pioglitazone is a thiazolidinedione anti-diabetic agent that decreases insulin resistance in the periphery and in the liver resulting in increased insulin-dependent glucose disposal and decreased hepatic glucose output [13]. On the other hand, Atorvastatin is a selective, competitive HMG-CoA reductase inhibitor which is primarily used to lower cholesterol and triglycerides in patients with hypercholesterolemia and mixed dyslipidemia [14].

\section{Materials and Methods}

\subsection{Animal Studies}

All protocols for the animal experiments were assessed and endorsed by the Animal Care and Use Committee of Institute of Biological Science, University of Rajshahi. Long-Evans male rats, weighed about 200 - 220 g, aged 2 months, were purchased from animal's house of International Centre for Diarrhoeal Disease Research, Bangladesh (ICDDRB). Prior to commencement of the experiments, all the rats were acclimatized to the new environmental condition for duration of one week. During the experimental period, the rats were kept in a well-ventilated animal house at room temperature and were supplied standard pellets from ICDDRB and fresh drinking water. All the rats were kept in protected cages and maintained with natural exposure to daylight and night. In both protocols (two weeks for short-term and four weeks for long-term), alloxan (120 mg/kg body weight, BW) was injected intra-peritoneally (i.p.) in rats. At first we treated alloxan $(120 \mathrm{mg} / \mathrm{kg} \mathrm{BW})$ induced diabetic rats with single dose of pioglitazone (10 mg/70 kg BW) and atorvastatin (20 mg/70 kg BW) for two weeks. Then we injected fixed dose combinations of pioglitazone (5 mg/70 kg BW) and atorvastatin (10 mg/70 kg BW) along with those of two drugs alone for four weeks. 


\subsection{Measurement of Glucose Level}

Pioglitazone, atorvastatin and combination of both were administered daily in alloxan-induced diabetic rats for both protocols. After two and four weeks treatment with drugs, blood glucose level was determined two hours after last dose, using Glucometer (One Touch Horizon, USA).

\subsection{Measurement of Lipid Profile}

After carrying out the treatment with proposed drugs, the rats were first anesthetized with sodium phenobarbital. Then abdominal skin was cut and thoracic artery was opened. Then 3 - $5 \mathrm{ml}$ of blood was collected directly from thoracic artery by syringes and centrifuged at $4000 \mathrm{rpm}$ for 10 minutes to obtain serum. Finally, serum lipid profile such as total cholesterol (TC), triglyceride (TG), low density lipoprotein-cholesterol (LDL-C) and high density lipoprotein-cholesterol (HDL-C) was investigated using diagnostic kits (Human, Germany).

\subsection{Evaluation of Free Radical Scavenging Activity}

The antioxidant activity using the 1,1-diphenyl-2-picrylhydrazyl (DPPH) was assessed as described previously [15]. The DPPH assay was performed by adding a sample (20 $\mu \mathrm{l}$ of blood plasma) plus $10 \mathrm{mM}$ sodium phosphate buffer (pH 7.4) to $400 \mu \mathrm{l}$ of $0.1 \mathrm{mM}$ methanol solution of DPPH. After $30 \mathrm{~min}$ incubation at ambient temperature $\left(21^{\circ} \mathrm{C}\right)$, absorbance of the samples at $517 \mathrm{~nm}$ was measured. As precipitate was formed in most of the plasma samples, they were centrifuged before the measurement.

\subsection{Estimation of SOD and Catalase in Liver Tissue}

After the experimental period, each animal was sacrificed; liver was isolated and homogenized in chilled tris buffer at a concentration of $10 \%(\mathrm{w} / \mathrm{v})$. The homogenate was centrifuge at $4000 \mathrm{rpm}$ for 15 minutes in cold centrifuge and supernatant was assayed for SOD and catalase activity. The catalase activity was assayed by the method described by Sinha (1972) [16]. SOD activity was determined by the method of Misra and Fridovich, 1972 [17].

\subsection{Histopathological Analysis}

Histological studies of pancreas were performed for the investigation of pancreatic beta cell recovery for the determination of LV weight and cardiomyocyte cross sectional area as previously reported procedure [18] [19] In brief, left ventricular (LV) weight was measured, and the ratio of LV weight to body weight was calculated to determine an index of LVH. One section was obtained from each pancreas, and four sections were obtained from each heart and then mounted on slides and stained with haematoxyline and eosin. To evaluate the extent of pancreacyte recovery and extent of cardiomyocyte hypertrophy, the cross-sectional images of pancreas and heart were scanned at $400 \times$ magnifications. Average values for each pancreas and heart were used for analysis. All images were taken using an Olympus BX51TF (Olympus Corporation, Tokyo, Japan).

\subsection{Statistical Analysis}

The results were expressed as mean \pm SEM. We used a one-way analysis of variance (ANOVA), followed by Dunnett's post-hoc test or students paired or unpaired $t$-test where appropriate. The statistical method applied in each analysis was described in each figure. Results were considered to be significant when $\mathrm{p}$ values were less than $0.05(\mathrm{p}<0.05)$.

\section{Results}

The parameters of blood glucose level, TC, TG, LDL, HDL, liver glycogen, antioxidant property, SOD and catalase enzyme activity were investigated and finally histology of heart and pancreas was demonstrated to study the effects of pioglitazone and atorvastatin alone and in combination on alloxan-induced diabetes and cardiac remodeling in rats using pioglitazone and atorvastatin as standard antidiabetic agent and lipid lowering agent respectively. 


\subsection{Effects of Pioglitazone and Atorvastatin on Blood Glucose Level in Short-Term AIDRs}

In contrast to normal rats ( $5 \mathrm{mmol} / \mathrm{lit})$, short-term i.p. injection of alloxan in AIDRS significantly increased blood glucose level (16 mmol/lit) (Figure 1(a)). To elucidate the individual effect of pioglitazone and atorvastatin on blood glucose level, we measured blood glucose level after two weeks treatment of pioglitazone and atorvastatin on AIDRs. Pioglitazone alone noticeably decreased blood glucose level from $16.03 \pm 0.90$ to $7.37 \pm$ $0.66 \mathrm{mmol} / \mathrm{lit}$, whereas atorvastatin alone failed to reduce significant amount of blood glucose level when compared with AIDRs (Figure 1(a)).

\subsection{Effects of Pioglitazone and Atorvastatin on Lipid Profile in Short-Term AIDRs}

In comparison with normal rats, significant fluctuation in lipid profile was observed after short-term induction of diabetes by alloxan (Figures 1(b)-(e)). To clarify the individual effect of pioglitazone and atorvastatin on lipid profile, we assayed TC, TG, LDL-C and HDL-C level after two weeks treatment of pioglitazone and atorvastatin in AIDRs. We found, both pioglitazone and atorvastatin reduced TC level 4.78\% and 38.64\% (Figure 1(b)), TG level 18.65\% and 30.98\% (Figure 1(c)), and LDL-C level 5.68\% and 26.02\% (Figure 1(d)), whereas both drug raised HDL-C level up to $30.95 \%$ and $41.73 \%$ (Figure 1(e)) correspondingly in contrast to diabetic rats. These results suggested that atorvastatin but not pioglitazone acted as lipid lowering agents. We also carried out histological analysis in AIDRs but did not notice any change in the LV weight and cardiomyocyte size.

\subsection{Effects of Combination Therapy on Blood Glucose Level in Long-Term AIDRs}

In case of long-term AIDRs, approximately two times increment in blood glucose level (32 mmol/lit) was observed
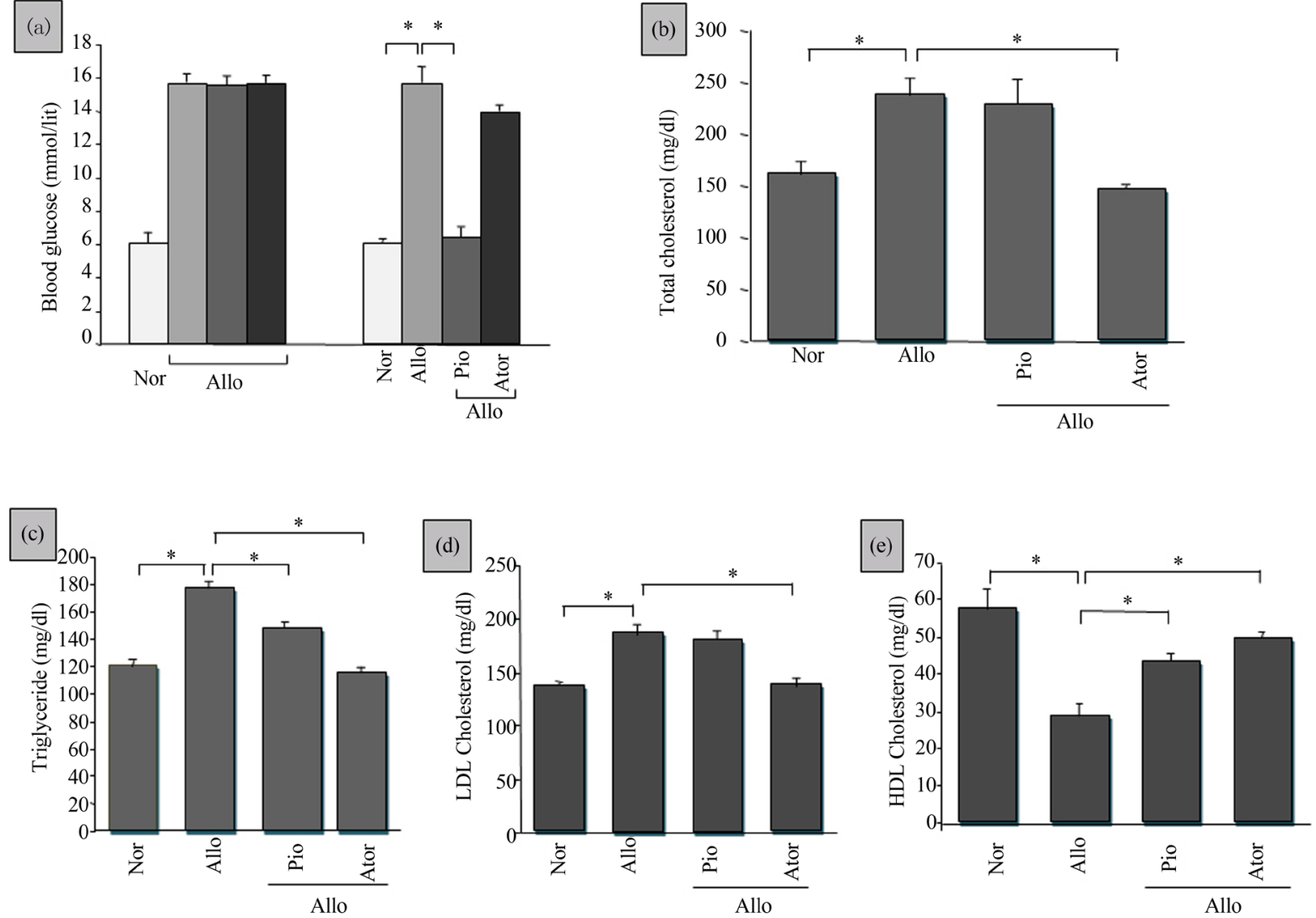

Figure 1. Observed effects of pioglitazone and atorvastatin for two weeks on blood glucose level and lipid profile in short-term AIDRs. (a) Blood glucose level; (b)-(e) TC, TG, LDL and HDL-C levels, respectively. All values were presented as mean \pm SEM; $n=6$ in each group, ${ }^{*} \mathrm{p}<0.05$ compared to AIDRs (ANOVA followed by Dunnett's test). Nor = Normal, Allo $=$ Alloxan, Pio $=$ Pioglitazone, Ator $=$ Atorvastatin . 
in comparison with short-term AIDRs (16 mmol/lit) (Figure 1(a) vs Figure 2(a)). Pioglitazone alone and combination of both significantly reduced blood glucose level from $31.54 \pm 2.19$ to $6.63 \pm 0.25 \mathrm{mmol} / \mathrm{lit}$ and $31.54 \pm$ 2.19 to $5.38 \pm 0.56 \mathrm{mmol} / \mathrm{lit}$, respectively when compared with diabetic rats after four weeks treatment. Atorvastatin failed to decrease the blood glucose level considerably when compared with diabetic rats (Figure 2(a)). Our results suggested that the combination therapy more effectively reduced the blood glucose level than that of mono-therapy in long-term AIDRs.

\subsection{Effects of Combination Therapy on Lipid Profile in Long-Term AIDRs}

It was found that pioglitazone, atorvastatin and combination of both reduced TC level $4.52 \%, 20.65 \%$ and $28.97 \%$ (Figure 2(b)), TG level 23\%, 32.4\% and 39.81\% (Figure 2(c)), and LDL-C level 5.02\%, 26.92\% and 37.16\% (Figure 2(d)) and increased HDL-C level 30.20\%, 37.58\% and 46.87\% (Figure 2(e)), respectively when compared with diabetic rats after four weeks treatment. In contrast, the effect of combination therapy on lipid profile was higher than that of mono-therapy alone. Pioglitazone did not show significant effect on lipid profile (Figures 2(b)-(e)). These results demonstrated that the combination therapy was more effective in lipid profile than that of mono-therapy in long-term AIDRs.

\subsection{Effects of Combination Therapy on DPPH Free Radical Scavenging Activity on Long-Term AIDRs}

After four weeks treatment with pioglitazone, atorvastatin and combination of both, it was observed that DPPH radical scavenging activity was increased $32.83 \%, 65.65 \%$ and $76.09 \%$, respectively in comparison with normal control group (Figure 3(a)). This result revealed that the combination therapy showed better antioxidant activity than mono-therapy in long-term AIDRs.
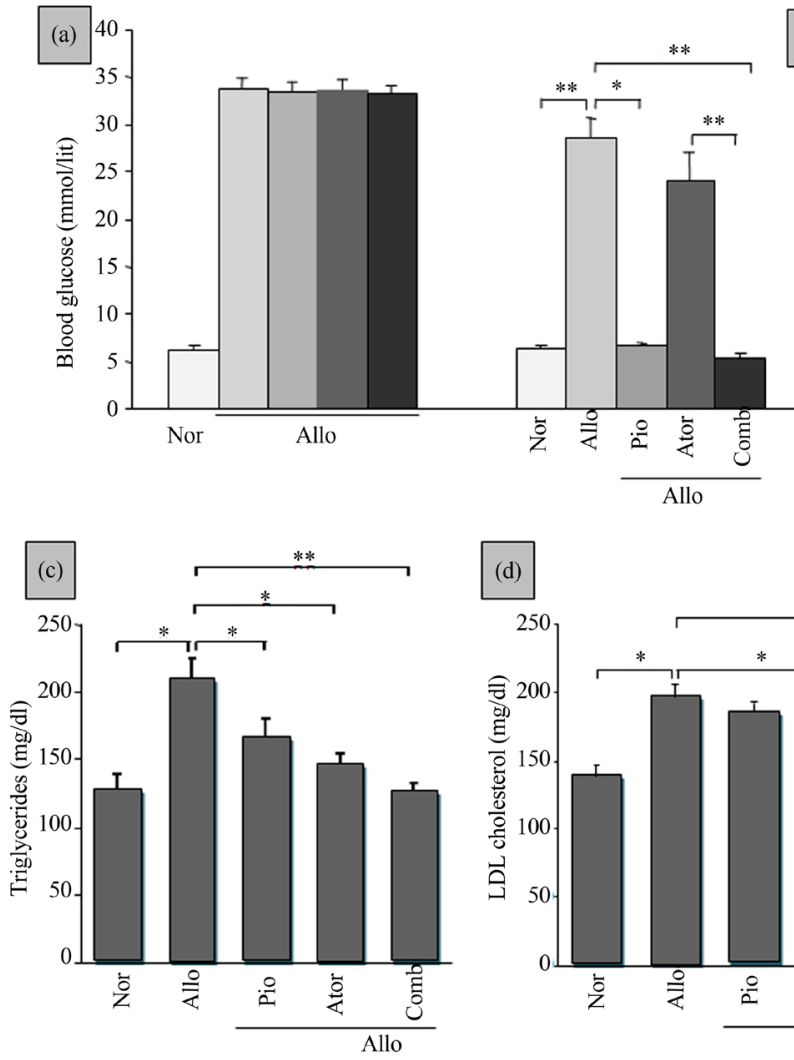
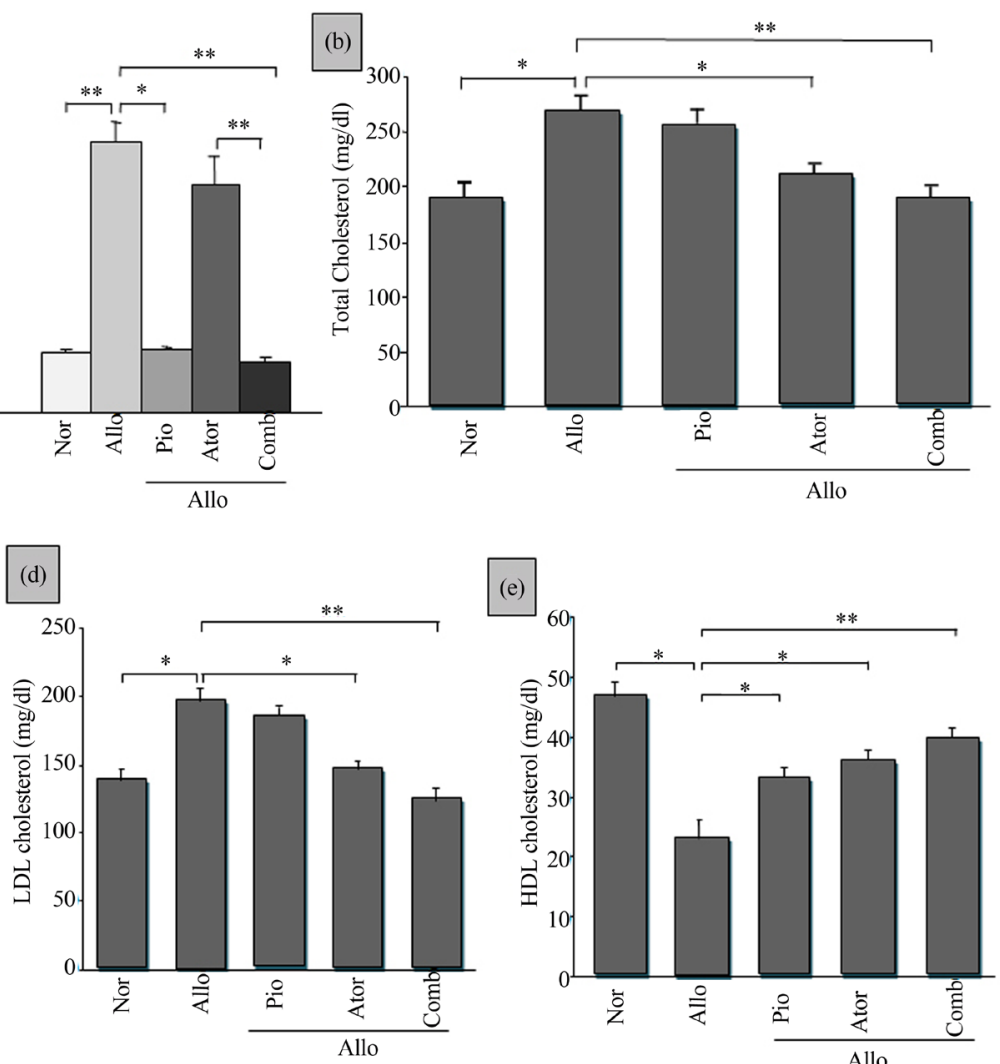

Figure 2. Observed effects of pioglitazone alone, atorvastatin alone and combination of both for four weeks on blood glucose level and lipid profile in long-term AIDRs. (a) Effect on blood glucose level; (b)-(e) TC, TG, LDL and HDL-C levels, respectively. Data were presented as mean \pm SEM; $n=6$ in each group, ${ }^{*} p<0.05$ and ${ }^{* *} p<0.01$ compared to AIDRs. Com = combination (Pioglitazone and atorvastation). 


\subsection{Effects of Drugs and Combination Therapy on SOD and Catalase Enzyme Activity}

The effects of pioglitazone, atorvastatin and combination of both on SOD (Figure 3(b)) and catalase (Figure 3(c)) enzyme in AIDRs were measured. After four weeks treatment with pioglitazone, atorvastatin and combination of both, it was observed that SOD and Catalase enzyme activity was increased 75\%, 85.25\%, 90.69\% and $15.36 \%, 34.61 \%, 57.69 \%$, respectively in comparison with diabetic control group. This outcome suggested that the combination therapy showed improved antioxidant activity than that of mono-therapy in long-term AIDRs.

\subsection{Effect of Drugs and Combination Therapy on Pancreas Islets of Langerhans Morphology}

Injection of alloxan in rats for four weeks significantly shrunk islets of Langerhans in comparison with normal rats. Treatment with pioglitazone and atorvastatin significantly recovered islets of Langerhans from shrinkage in AIDRs. But in case of combination therapy, noticeable recovery of Langerhans cell was reported (Figure 4).

\subsection{Effects of Combination Therapy on LV Hypertrophy on Long-Term AIDRs}

Induction of diabetes by alloxan for four weeks in rats significantly increased LV hypertrophy (0.00416) when compared with normal rats (0.00274). We examined the effect of single drug and combination of both on LV hypertrophy (LV weight/body weight) in long-term AIDRs to know whether or not the combination therapy was more effective than mono-therapy alone. After four weeks treatment, atorvastatin alone and the proposed combination therapy significantly reduced LVH ( 0.00341 and 0.00310 respectively) when compared with diabetic rats whereas the combination therapy reduced LVH more effectively than mono-therapy (Figure 5). On the other hand, pioglitazone alone did not significantly reduce LV hypertrophy (0.00395) when compared with diabetic rats.

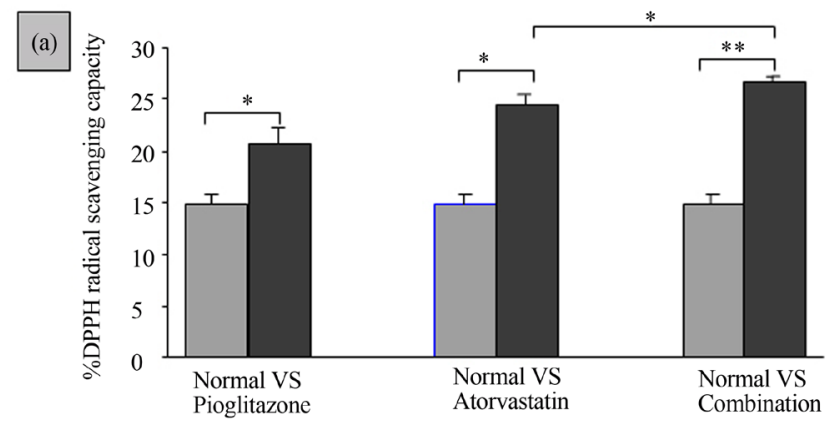

(b)
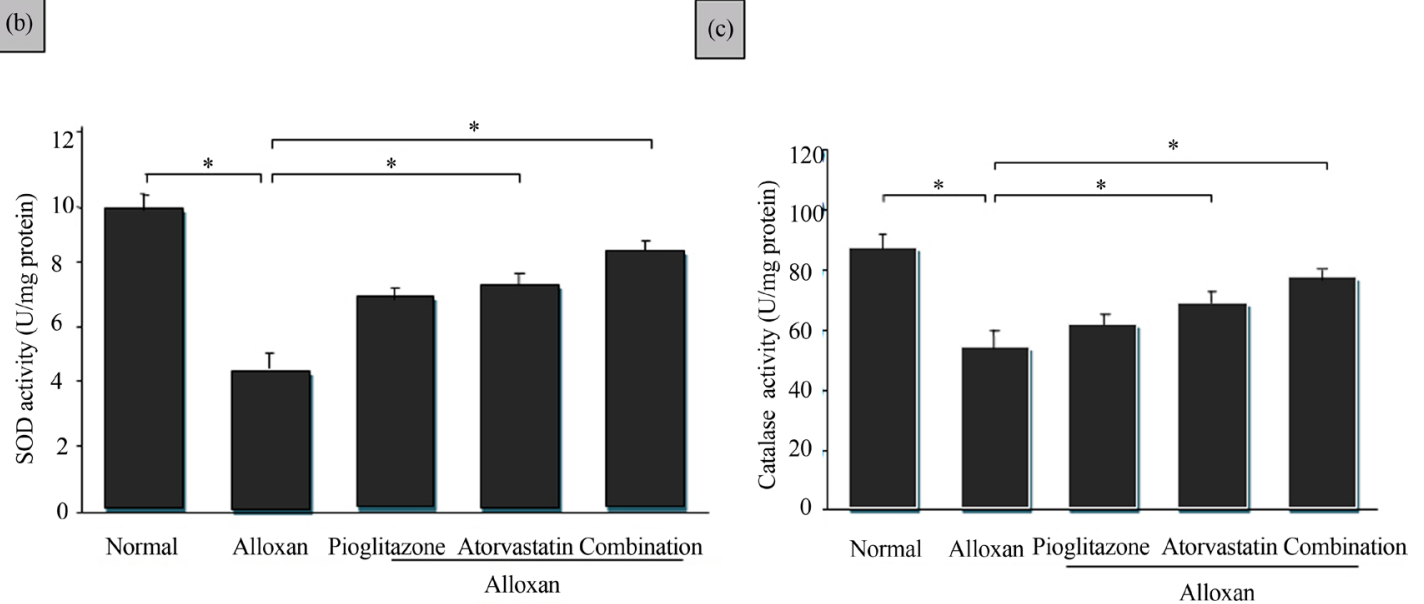

Figure 3. Observed effects of Pioglitazone alone, atorvastatin alone and combination of both on (a) DPPH radical scavenging activity; (b) SOD enzyme activity; and (c) Catalase activity in long-term AIDRs. The data were shown as mean \pm SEM, $n=6$ in each case. ${ }^{*} \mathrm{p}<0.05$ and ${ }^{* *} \mathrm{p}<0.01$ compared to long term AIDRs (ANOVA followed by Dunnett's test). 


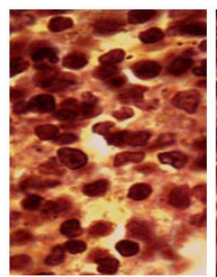

Normal

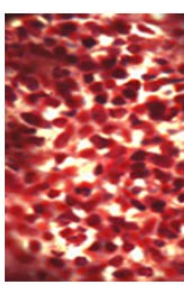

Alloxan

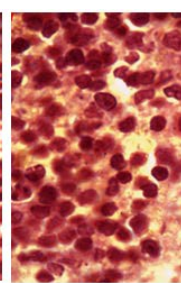

Pioglitazone

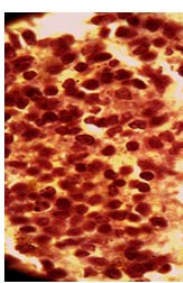

Atorvastatin

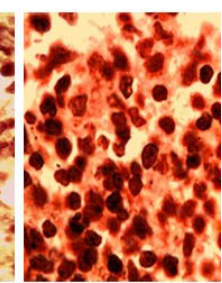

Combination

Alloxan

Figure 4. Microscopic view of pancreatic islets of Langerhans cells at $400 \times$ magnification after four weeks' treatment with pioglitazone, atorvastatine and their combination in AIDRs.

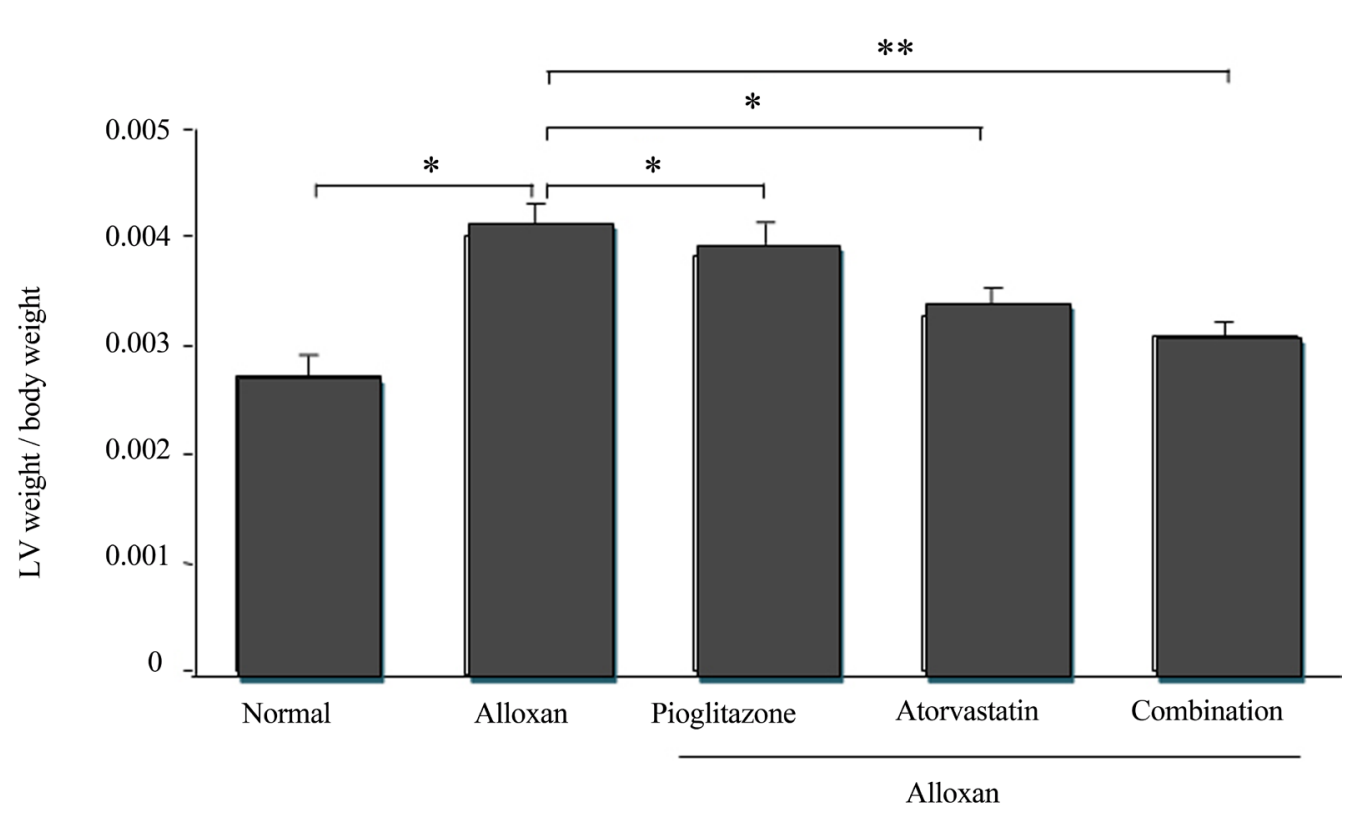

Figure 5. Observed effects of pioglitazone, atorvastatin and their combination on LV hypertrophy. The data were shown as mean \pm SEM, $n=6$ in each case. ${ }^{*} \mathrm{p}<0.05$ and ${ }^{* *} \mathrm{p}<0.01$ vs long term AIDRs (ANOVA followed by Dunnett's test).

\section{Discussion}

Diabetes is a great threat to public health globally that is getting worse rapidly, having the biggest impact is on adults of working age in developing countries. In most developing countries at least one in ten deaths in adults, aged between 35 to 64 is attributable to diabetes and in some the figure is as high as one in five [20].

Diabetes can be controlled successfully and the risk of developing secondary complications can be reduced significantly. In order to do so, scientists and researchers are engaged in research to find out new drug combination therapy and natural plant products to combat diabetes with CVD worldwide.

The result of the present study indicated that pioglitazone lowered blood glucose level in AIDRs significantly whereas atorvastatin did not produce any considerable change of this parameter which supported the findings of earlier published report [21] [22]. Here we observed that the combination therapy efficiently controlled diabetes in comparison with pioglitazone alone in long-term AIDRs. However, investigation should continue to elucidate the exact mechanism of this effect, offered by combination therapy.

It had been reported that alloxan treatment not only increased blood glucose levels but also increased the levels of TG, TC, and LDL cholesterol and decreased the levels of HDL cholesterol in diabetic rats [23] [24]. Factors which influence the glucose metabolism under various physiological conditions, can influence lipid metabolism as well [25]. It had also been reported that TG accumulation increased considerably in diabetes mellitus 
[26]. Hypercholesterolemia and hypertriglyceridemia had been reported to occur in diabetes [27].

In our study, it was found that the TC, TG, LDL-Cholesterol level were reduced and HDL-Cholesterol level were increased in AIDRs in comparison with normal rats after the both two and four weeks treatment. The serum TC, TG, LDL-C levels were significantly decreased and HDL-C level was markedly increased after two weeks treatment with atorvastatin in comparison with AIDRs. We also found similar results on lipid profile after four weeks treatment with atorvastatin alone and combination therapy (pioglitazone and atorvastatin). Interestingly, the combination therapy was more effective than atorvastatin alone. Also, pioglitazone alone exhibited negligible effect on lipid profile in both two and four week's studies. Our findings were in consistence with the previously published data [21] [22]. For the distinct lipid lowering capacity of this combination therapy, it might be proposed that the drugs of the combination therapy may act as inhibitors for enzymes, such as hydroxyl-methyl-glutaryl-CoA reductase which participates in de novo cholesterol biosynthesis.

It had been established that hyperglycemia promotes oxidative stress and oxidative stress further potentiates secondary CVD [28]. Free radicals can injure the cardinal cellular components such as lipids, proteins, and nucleic acids (e.g. DNA), leading to subsequent cell death. The damage can become more prevalent due to weakened cellular antioxidant defense systems. In our study, it was observed that treatment with pioglitazone, atorvastatin and combination therapy significantly increased DPPH radical scavenging activity in long-term AIDRs. However, combination therapy showed higher antioxidant properties than that of alone. Current study suggests that atorvastatin together with pioglitazone might be more efficient for alleviating oxidative stress associated with diabetes in rats.

Earlier studies established that diabetic rats usually lack SOD and catalase enzyme activity [29]. To investigate the beneficial effects of combination therapy (pioglitazone and atorvastatin), we further examined these enzymes' activity in AIDRs after four weeks. Current research revealed that long term induction of alloxan in rats significantly decreased antioxidant enzyme (SOD and catalase) activity in comparison with normal rats. Treatment with combination therapy significantly increased these enzymes' activity in AIDRs. Atorvastatin alone also significantly increased antioxidant enzyme (SOD and catalase) activity whereas combination therapy showed better effect than that of atorvastatin alone.

In case of long term AIDRs, islets of Langerhans cells shrunk markedly in comparison with normal rats. Administration of pioglitazone as well as atorvastatin recovered Langerhans cells from shrinkage to a certain extent which was demonstrated earlier [30]. In our present investigation we observed that treatment with combination therapy almost turns back shrinkage of Langerhans cell.

Previous studies showed that long-term induction of diabetes without any intervention of hypolipidemic agent produced CVD [31]. In our current investigation, induction of alloxan in rats for four weeks significantly $(\mathrm{p}<$ 0.05 ) increased LV hypertrophy in comparison with normal rats. Treatment with combination therapy (pioglitazone and atorvastatin) noticeably $(\mathrm{p}<0.01$ ) reduced LV hypertrophy in long term AIDRs. Atorvastatin alone also significantly reduced LV hypertrophy whereas combination therapy had a greater impact than atorvastatin alone in this protocol.

\section{Conclusion}

Findings of current investigation evidently established that the proposed combination therapy possessed hypoglycemic, hypolipidemic and antioxidant activities while AIDRs were employed as animal model for diabetes mellitus. Thus, this therapy might suggest the use of such effective combination of drugs (pioglitazone and atorvastatin) in the treatment of diabetes and related CVDs. Proposed medication may also append to the development of new prescription drug for the treatment of diabetes with its risk factors. Although this study gave some of the introductory concepts for effective management of blood glucose level and lipid profile with conventional medicine, further study is still required for assessing tolerability, pharmacodynamic and pharmacokinetic properties as well as mechanism of action of this fixed dose combination therapy (pioglitazone and atorvastatin) which might disclose new remedies for the treatment of diabetes with cardiovascular diseases.

\section{Acknowledgements}

The authors thank Square Pharmaceuticals Ltd, Bangladesh for its kind gift of atorvastatin and pioglitazone. This work was carried out as part of M. Pharm study with financial support from the Department of Pharmacy, University of Rajshahi, Bangladesh and NSICT (National Science and Information \& Communication Technol- 
ogy) fellowship, Bangladesh.

\section{Conflict of Interest}

The authors declare that they have no conflict of interest to disclose.

\section{References}

[1] Sharma, V.K., Kumar, S., Patel, H.J., et al. (2010) Hypoglycemic Activity of Ficus Glomerata in Alloxan Induced Diabetic Rats. International Journal of Pharmaceutical Sciences Review and Research, 1, 18-22.

[2] World Health Organization (2011) Global Status Report on Noncommunicable Diseases 2010. World Health Organization, Geneva.

[3] Wild, S., Roglic, G., Green, A., et al. (2004) Global Prevalence of Diabetes: Estimates for 2000 and Projections for 2030. Diabetes Care, 27, 1047-1053.

[4] Kumar, P. and Clark, M. (2002) Diabetes Mellitus and Other Disorders of Metabolism. In: Sunders, W.B., Ed., Clinical Medicine, Vol. 2, Elsevier, London, 1069-1071.

[5] Amira, A.M.A. (2010) Oxidative Stress and Disease: An Update Review. Research Journal of Immunology, 3, 129145. http://dx.doi.org/10.3923/rji.2010.129.145

[6] Vijaya, L.S.V., Padmaja, G., Periannan, K., et al. (2009) Oxidative Stress in Cardiovascular Disease. Indian Journal of Biochemistry and Biophysics, 46, 421-440.

[7] Hsieh, C.C., Yen, M.H., Yen, C.H., et al. (2001) Oxidised Low Density Lipoprotein Induce Apoptosis via Generation of Reactive Oxygen Species in Vascular Smooth Muscle Cells. Cardiovascular Research, 49, 135-145. http://dx.doi.org/10.1016/S0008-6363(00)00218-2

[8] Phan, S.H., Gannon, D.E., Varani, J., et al. (1989) Xanthine Oxidase Activity in Rat Pulmonary Artery Endothelial Cells and Its Alteration by Activated Neutrophils. The American Journal of Pathology, 134, 1201-1211.

[9] Akihiro, M., Yoshitaka, H., Hiroaki, S., et al. (2001) Possible Involvement of Rho-Kinase in the Pathogenesis of Hypertension in Humans. Hypertension, 38, 1307-1310. http://dx.doi.org/10.1161/hy1201.096541

[10] Malviya, N., Jain, S. and Malvia, S. (2010) Antidiabetic Potential of Medicinal Plants. Acta Poloniae Pharmaceutica, 67, 113-138.

[11] Matafome, P., Louro, T., Rodriques, L., et al. (2011) Metformin and Atorvastatin Combination Further Protect the Liver in Type 2 Diabetes with Hyperlipidaemia. Diabetes/metabolism Research and Reviews, 27, 54-62. http://dx.doi.org/10.1002/dmrr.1157

[12] Pieroni, A., Nebel, S., Quave, C., et al. (2002) Ethnopharmacology of Liakra: Traditional Weedy Vegetables of the Arbëreshë of the Vulture Area in Southern Italy. Journal of Ethnopharmacology, 81, 165-185. http://dx.doi.org/10.1016/S0378-8741(02)00052-1

[13] Yates, S.W. (2004) Comparative Effects of Available Thiazolidinediones: A Review of the Literature. P\&T, 29, 584-588.

[14] Grundy, S.M. (1988) HMG-CoA Reductase Inhibitors for Treatment of Hypercholesterolemia. New England Journal of Medicine, 319, 24-33. http://dx.doi.org/10.1056/NEJM198807073190105

[15] Blois, M.S. (1958) Antioxidant Determinations by the Use of a Stable Free Radical. Nature, 181, 1199-1200. http://dx.doi.org/10.1038/1811199a0

[16] Sinha, A.K. (1972) Colorimetric Assay of Catalase. Analytical Biochemistry, 47, 389-394. http://dx.doi.org/10.1016/0003-2697(72)90132-7

[17] Misra, H.P. and Fridovich, I. (1972) The Role of Superoxide Anion in the Autoxidation of Epinephrine and a Simple Assay for Superoxide Dismutase. Journal of Biological Chemistry, 247, 3170-3175.

[18] Albajali, A.A., Nagi, A.H., Shahzad, M., et al. (2011) Effect of Allium Sativa L. on Pancreatic B Cells in Comparison to Nigella Sativa L. in Streptozotocin Induced Diabetic Rats. Journal of Medicinal Plants Research, 5, 5779-5784.

[19] Higashi, M., Shimokawa, H., Hattori, T., et al. (2003) Long-Term Inhibition of Rho-Kinase Suppresses Angiotensin II-Induced Cardiovascular Hypertrophy in Rats in Vivo: Effect on Endothelial NAD(P)H Oxidase System. Circulation Research, 93, 767-775. http://dx.doi.org/10.1161/01.RES.0000096650.91688.28

[20] (2004) Source: "Diabetes Action Now.” An initiative of the World Health Organization and the International Diabetic Federation. WHO, Geneva, IDF, Brussels, 1-20.

[21] Balasubramanian, R., Varadharajan, S., Kathale, A., et al. (2008) Assessment of the Efficacy and Tolerability of a Fixed Dose Combination of Atorvastatin $10 \mathrm{mg}+$ Metformin SR $500 \mathrm{mg}$ in Diabetic Dyslipidaemia in Adult Indian 
Patients. Journal of the Indian Medical Association, 106, 464-467.

[22] Babu, V., Gangadevi, T. and Subramanium, A. (2002) Antihyperglycemic Activity of Cassia kleinii Leaf Extract in Glucose Feed Normal Rats and Alloxan Induced Diabetic Rats. Indian Journal of Pharmacology, 34, 409-415.

[23] Trivedi, N.A., Mazumdar, B., Bhatt, J.D., et al. (2004) Effect of Shilajit on Blood Glucose and Lipid Profile in Alloxan Induced Diabetic Rats. Indian Journal of Pharmacology, 36, 373-376.

[24] Jenkins, D.J.A., Jenkins, A.I., Wolever, T.M.S., et al. (1995) Effect of Reduced Rat of Carbohydrate Absorption on Carbohydrate and Lipid Metabolism. European Journal of Clinical Nutrition, 49, 68-73.

[25] Iams, S.G. and Wexler, B.C. (1977) Alloxan Diabetes in Spontaneously Hypertensive Rats: Gravimetric, Metabolic and Histopathological Alterations. British Journal of Experimental Pathology, 58, 177-199.

[26] Riyad, A., Abdul, G.A.S. and Suleiman, S.M. (1988) Effect of Fenugreek and Lupine Seeds on the Development of Experimental Diabetes in Rats. Planta Medica, 54, 286-290. http://dx.doi.org/10.1055/s-2006-962434

[27] Sharma, S.R., Dwivedi, S.K. and Swarup, D. (1996) Hypoglycaemic and Hypolipidaemic Effects of Cinnamomum Tamala Nees Leaves. Indian Journal of Experimental Biology, 34, 372-374.

[28] Munzel, T., Gori, T., Bruno, R.M. and Taddei, S. (2010) Is Oxidative Stress a Therapeutic Target in Cardiovascular Disease? European Heart Journal, 31, 2741-2749. http://dx.doi.org/10.1093/eurheartj/ehq396

[29] Patel, T.B., Patel, L.D., Patel, T.B., et al. (2010) Effects of Atorvastatin as Antioxidants in Diabetic Associated Cardiovascular Complications. Journal of Pharmaceutical Sciences and Research, 2, 247-256.

[30] Campbell, I.W. (2006) The Role of Metformin and Pioglitazone in Early Combination Treatment: TZDs and Beta-Cell Preservation. British Journal of Diabetes and Vascular Disease, 6, 207-215. http://dx.doi.org/10.1177/14746514060060050101

[31] Tsutsui, H., Kinugawa, S., Matsushima, S., et al. (2011) Oxidative Stress in Cardiac and Skeletal Muscle Dysfunction Associated with Diabetes Mellitus. Journal of Clinical Biochemistry and Nutrition, 48, 68-71. http://dx.doi.org/10.3164/jcbn.11-012FR 